KYUNGPOOK Math. J. 53(2013), 639-646

http://dx.doi.org/10.5666/KMJ.2013.53.4.646

\title{
Approximately Orthogonal Additive Set-valued Mappings
}

Alireza Kamel Mirmostafaee* and Mostafa Mahdavi

Center of Excellence in Analysis on Algebraic Structures, Department of pure Mathematics, School of Mathematical Sciences, Ferdowsi University of Mashhad 91775, Iran

e-mail: mirmostafaei@um.ac.ir and m_mahdavi1387@yahoo.com

ABSTRACT. We investigate the stability of orthogonally additive set-valued functional equation

$$
F(x+y)=F(x)+F(y) \quad(x \perp y)
$$

in Hausdorff topology on closed convex subsets of a Banach space.

\section{Introduction}

A functional equation $\mathfrak{F}$ is called stable if for any function $f$ satisfying approximately to the equation $\mathfrak{F}$, there is a true solution of $\mathfrak{F}$ near to $f$. In 1940, S. M. Ulam [24] proposed the first stability problem for group homomorphisms. Hyers [9] gave the first significant partial solution to his problem for linear functions. Th. M. Rassias [20] improved Hyers' theorem by weakening the condition for the Cauchy difference controlled by $\|x\|^{p}+\|y\|^{p}, p \in[0,1)$. For some recent developments in this area, we refer the reader to the articles $[5,6,11,12,15,19]$ and the references therein.

In 1985, Rätz[21] gave a generalization of Birkhoff-James orthogonality [1, 10] in vector spaces. He also investigated some properties of orthogonally additive functional equation. This definition motivated some Mathematicians to discuss about the orthogonal stability of functional equations (see e. g. $[8,13,16,22]$ ). On the other hand, set-valued mappings and their stability have been investigated by some authors from different point of view $[2,7,14,17,23]$.

In the next section, we prove the stability of set-valued orthogonal additive functional equation

$$
F(x+y)=F(x)+F(y) \quad(x \perp y) .
$$

* Corresponding Author.

Received June 11, 2012; accepted August 30, 2012.

2010 Mathematics Subject Classification: 39B22, 39B55, 39B62, 39B82.

Key words and phrases: Set-valued mappings, orthogonal space, Hausdorff metric, HyersUlam stability.

This research was supported by a grant from Ferdowsi University of Mashhad No. MP91281 MIM. 
In fact, we will show if $(X, \perp)$ is an orthogonal space, $Y$ is a Banach space and $F: X \rightarrow C C(Y)$ is an even function such that

$$
\mathcal{H}(F(x+y), F(x)+F(y)) \leq \varepsilon \quad(x, y \in X, x \perp y),
$$

for some $\varepsilon>0$. Then there exists a unique quadratic function $Q: X \rightarrow C C(Y)$ such that

$$
\mathcal{H}(F(x), Q(x)) \leq \frac{7 \varepsilon}{4} \quad(x \in X) .
$$

In this case, we will show that there is a quadratic function $q: X \rightarrow Y$ such that

$$
q(x) \in F(x)+\frac{7 \varepsilon}{3} \overline{B(0,1)} \quad(x \in X) .
$$

\section{Main Results}

Throughout the paper, unless otherwise stated, we will assume that $X$ and $Y$ are topological vector spaces over $\mathbb{R}$. If $A, B \subset Y$ and $\lambda \in \mathbb{R}$, we use the following notions

$$
A+B=\{a+b: a \in A, \quad b \in B\}, \quad \lambda A=\{\lambda a: a \in A\} .
$$

The following properties will often be used in the sequel:

For each $A, B \subset Y$ and $\lambda, \mu \geq 0$, we have

$$
\lambda(A+B)=\lambda A+\lambda B, \quad(\lambda+\mu) A \subseteq \lambda A+\mu A .
$$

Moreover, if $A$ is convex, $(\lambda+\mu) A=\lambda A+\mu A$.

Definition 2.1. Let $Y$ be a normed space and $A_{1}, A_{2} \subseteq Y$ be non-empty closed bounded sets. Then the Hausdorff distance between $A_{1}$ and $A_{2}$ is defined by

$$
\mathcal{H}\left(A_{1}, A_{2}\right):=\inf \left\{s>0: A_{1} \subseteq A_{2}+s \overline{B(0,1)} \text { and } A_{2} \subseteq A_{1}+s \overline{B(0,1)}\right\} .
$$

It is known that $\mathcal{H}$ defines a metric on closed convex subsets of $Y$, which is called Hausdorff metric topology $[3,4]$. Moreover, if $Y$ is a Banach space, $(C C(Y), \mathcal{H})$, the space of all non-empty compact convex subsets of $Y$ with the Hausdorff metric topology is a complete metric space [3].

In 1985, Rätz [21] introduced the following notion:

Definition 2.2. Let $X$ be a real topological vector space of dimension $\geq 2$. A binary relation $\perp \subset X \times X$ is called an orthogonal relation if the following properties hold.

(1) $x \perp 0,0 \perp x$ for every $x \in X$, 
(2) if $x, y \in X \backslash\{0\}, x \perp y$, then $x$ and $y$ are linearly independent;

(3) if $x, y \in X, x \perp y, \alpha x \perp \beta y$ for all $\alpha, \beta \in \mathbb{R}$,

(4) if $P$ is a two dimensional subspace of $X, x \in P, \lambda \in \mathbb{R}^{+}$, then there exists some $y \in P$ such that $x \perp y$ and $x+y \perp \lambda x-y$.

The space $X$ with an orthogonal relation $\perp$ is called an orthogonally space and is denoted by $(X, \perp)$.

Definition 2.3. Let $X$ and $Z$ be two sets. A function $Q: X \rightarrow Z$ is called quadratic if $Q(x+y)+Q(x-y)=2 Q(x)+2 Q(y)$ for all $x, y \in X$.

We need to the following result due to Rådström [18].

Lemma 2.4. Let $A, B$ and $C$ be nonempty subsets of a topological vector space $Y$. Suppose that $B$ is closed and convex and $C$ is bounded. If $A+C \subseteq B+C$, then $A \subseteq B$. If moreover, $A$ is closed and convex and $A+C=B+C$, then $A=B$.

Now, we are ready to state the main result of this paper.

Theorem 2.5. Let $X$ be a topological vector space over $\mathbb{R}$ which is also an orthogonal space and let $Y$ be a Banach space. Let $F: X \rightarrow C C(Y)$ be an even function and for some $\varepsilon>0$,

$$
\mathcal{H}(F(x+y), F(x)+F(y)) \leq \varepsilon \quad(x, y \in X, x \perp y) .
$$

Then there exists a unique quadratic and orthogonal additive function $Q: X \rightarrow$ $C C(Y)$ such that

$$
\mathcal{H}(F(x), Q(x)) \leq \frac{7 \varepsilon}{3} \quad(x \in X) .
$$

Proof. We divide the proof into several steps.

Step 1.For each $x \in X$,

$$
\mathcal{H}(F(2 x), 4 F(x)) \leq 7 \varepsilon
$$

Proof of step 1. By Definition 2.2, for each $x \in X$, there is some $y \in X$ such that $x \perp y$ and $x+y \perp x-y$. Take some $y \in X$ with this property. Then 


$$
\begin{aligned}
F(x) & =F\left(\frac{x+y}{2}+\frac{x-y}{2}\right) \\
& \subseteq F\left(\frac{x+y}{2}\right)+F\left(\frac{x-y}{2}\right)+\varepsilon \overline{B(0,1)} \\
& =F\left(\frac{x+y}{2}\right)+F\left(\frac{y-x}{2}\right)+\varepsilon \overline{B(0,1)} \quad(\because F \text { is even }) \\
& \subseteq F\left(\frac{x+y}{2}+\frac{y-x}{2}\right)+2 \varepsilon \overline{B(0,1)} \\
& =F(y)+2 \varepsilon \overline{B(0,1)} .
\end{aligned}
$$

Since $x+y \perp y-x$, by interchanging the role of $x$ and $y$, we see that

$$
F(y) \subseteq F(x)+2 \varepsilon \overline{B(0,1)} .
$$

On the other hand,

$$
\begin{aligned}
F(2 x) & =F(x+y+x-y) \subseteq F(x+y)+F(x-y)+\varepsilon \overline{B(0,1)} \\
& \subseteq 2 F(x)+2 F(y)+3 \varepsilon \overline{B(0,1)} \\
& \subseteq 4 F(x)+7 \varepsilon \overline{B(0,1)}
\end{aligned}
$$

and

$$
\begin{aligned}
4 F(x) & =2 F(x)+2 F(x) \subseteq 2 F(x)+2 F(y)+4 \varepsilon \overline{B(0,1)} \\
& \subseteq F(x)+F(y)+F(x)+F(-y)+4 \varepsilon \overline{B(0,1)}(\text { since } x \perp y) \\
& \subseteq F(x+y)+F(x-y)+6 \varepsilon \overline{B(0,1)}(\text { since } x+y \perp x-y) \\
& \subseteq F(2 x)+7 \varepsilon \overline{B(0,1)} .
\end{aligned}
$$

Therefore (2.2) holds.

Step 2. There is a unique orthogonal additive function $Q: X \rightarrow C C(Y)$ such that

$Q(2 x)=4 Q(x)$ and

$$
\mathcal{H}(F(x), Q(x)) \leq \frac{7 \varepsilon}{3}
$$

for each $x \in X$.

Proof of step 2. Replace $x$ by $2^{n} x$ in (2.2) and multiply both sides of the obtained inequality by $4^{-(n+1)}$ to obtain the following inequality

$$
\mathcal{H}\left(4^{-(n+1)} F\left(2^{n+1} x\right), 4^{-n} F\left(2^{n} x\right)\right) \leq \frac{7 \varepsilon}{4^{n+1}} \quad(n \geq 0, x \in X) .
$$


It follows that for each $n>m \geq 0$, we have

$$
\begin{aligned}
\mathcal{H}\left(4^{-n} F\left(2^{n} x\right), 4^{-m} F\left(2^{m} x\right)\right) & \leq \sum_{k=m}^{n-1} \mathcal{H}\left(4^{-(k+1)} F\left(2^{k+1} x\right), 4^{-k} F\left(2^{k} x\right)\right) \\
& \leq \sum_{k=m}^{n-1} \frac{7 \varepsilon}{4^{k+1}} \quad(x \in X) .
\end{aligned}
$$

Since the right hand side of the above inequality tends to zero as $n \rightarrow \infty$, $\left\{4^{-n} F\left(2^{n} x\right)\right\}$ is a Cauchy sequence in $(C C(Y), \mathcal{H})$. Completeness of $C C(Y)$ with respect to the Hausdorff metric topology insures that

$$
Q(x)=\lim _{n \rightarrow \infty} 4^{-n} F\left(2^{n} x\right) \quad(x \in X)
$$

defines a function from $X$ to $C C(Y)$. Put $m=0$ in (2.4) to obtain

$$
\begin{aligned}
\mathcal{H}(Q(x), F(x)) & =\lim _{n \rightarrow \infty} \mathcal{H}\left(4^{-n} F\left(2^{n} x\right), F(x)\right) \\
& \leq \sum_{k=0}^{\infty} \frac{7 \varepsilon}{4^{k+1}}=\frac{7 \varepsilon}{3} \quad(x \in X) .
\end{aligned}
$$

Moreover, for every $x \in X$, we have

$$
\begin{aligned}
Q(2 x) & =\lim _{n \rightarrow \infty} 4^{-n} F\left(2^{n+1} x\right) \\
& =4 \lim _{n \rightarrow \infty} 4^{-(n+1)} F\left(2^{n+1} x\right)=4 Q(x) .
\end{aligned}
$$

If $x \perp y$, we have

$$
\begin{aligned}
& \mathcal{H}(Q(x)+Q(y), Q(x+y)) \\
& =\lim _{n \rightarrow \infty} \mathcal{H}\left(4^{-n} F\left(2^{n} x\right)+4^{-n} F\left(2^{n} y\right), 4^{-n} F\left(2^{n}(x+y)\right)\right) \leq \lim _{n \rightarrow \infty} 4^{-n} \varepsilon=0 .
\end{aligned}
$$

Hence $Q$ is orthogonal additive. Suppose that $Q^{\prime}: X \rightarrow C C(Y)$ satisfies the following properties:

(i) $\mathcal{H}\left(Q^{\prime}(x), F(x)\right) \leq \frac{7 \varepsilon}{3}$ and

(ii) $Q^{\prime}(2 x)=4 Q^{\prime}(x)$ for each $x \in X$.

Then for each $x \in X$, we have

$$
\begin{aligned}
\mathcal{H}\left(Q^{\prime}(x), Q(x)\right) & =\lim _{n \rightarrow \infty} \mathcal{H}\left(4^{-n} Q^{\prime}\left(2^{n} x\right), 4^{-n} F\left(2^{n} x\right)\right) \\
& =\lim _{n \rightarrow \infty} 4^{-n} \mathcal{H}\left(Q^{\prime}\left(2^{n} x\right), F\left(2^{n} x\right)\right) \leq \lim _{n \rightarrow \infty} 4^{-n} \frac{7 \varepsilon}{3}=0 .
\end{aligned}
$$

Thus the uniqueness assertion of step 2 follows.

Step 3. The function $Q: X \rightarrow C C(Y)$ is quadratic. 
Proof of step 3. Let $x, y \in X$. Then the following cases may happen.

(i) $y=\alpha x$, where $\alpha \geq 0$. In this case, by property (4) of Definition 2.2, for each $x \in X$, there is some $z \in X$ such that $x \perp z$ and $x+z \perp \alpha x-z$. Therefore

$Q(x+y)+Q(x-y)=Q(x+\alpha x)+Q(x-\alpha x)=Q(x+z+\alpha x-z)+Q(\alpha x-x)$.

It follows that

$$
\begin{aligned}
Q(x+\alpha x)+Q(x-\alpha x)+Q(2 z) & =Q(x+z)+Q(\alpha x-z)+Q(\alpha x-x+2 z) \\
& =Q(x)+2 Q(z)+Q(\alpha x)+Q(x+z+z-\alpha x) \\
& =Q(x)+2 Q(z)+Q(\alpha x)+Q(x+z)+Q(z-\alpha x) \\
& =2 Q(x)+2 Q(\alpha x)+4 Q(z) \\
& =2 Q(x)+2 Q(\alpha x)+Q(2 z) .
\end{aligned}
$$

Thanks to Lemma 2.4, the result follows in this case.

(ii) $y=\alpha x$, where $\alpha<0$. Let $\beta=-\alpha$. Then $\beta>0$. Hence,

$$
\begin{aligned}
Q(x+\alpha x)+Q(x-\alpha x) & =Q(x-\beta x)+Q(x+\beta x) \\
& =2 Q(x)+2 Q(\beta x)=2 Q(x)+2 Q(\alpha x)
\end{aligned}
$$

since $Q$ is even.

(iii) $x$ and $y$ are linearly independent.

By Definition 2.2, there is some $z$ in linear span of $\{x, y\}$ such that $x \perp z$. Let $y=\alpha x+\beta z$. Then

$$
\begin{aligned}
Q(x+y)+Q(x-y) & =Q[(x+\alpha x)+\beta z]+Q[x-(\alpha x+\beta z)] \\
& =Q(x+\alpha x)+Q(\beta z)+Q(x-\alpha x)+Q(-\beta z) \\
& =2 Q(x)+2 Q(\alpha x)+2 Q(\beta z) \\
& =2 Q(x)+2 Q(\alpha x+\beta z)=2 Q(x)+2 Q(y) .
\end{aligned}
$$

This completes the proof of the theorem.

Example 2.6. Let $X$ be an inner product space and $\varepsilon>0$. Define $F: X \rightarrow C C(\mathbb{R})$ by $F(x)=\left[0,\|x\|^{2}+\varepsilon\right]$. It is easy to see that $F$ is $[0, \varepsilon]$-orthogonal additive even function. According to Theorem 2.5, there is a quadratic function $Q: X \rightarrow C C(\mathbb{R})$ such that

$$
\mathcal{H}(F(x), Q(x)) \leq \frac{7 \varepsilon}{3} \quad(x \in X) .
$$

Definition 2.7. Let $X$ and $Y$ be two sets. By a selection of a set-valued function $F: X \rightarrow 2^{Y}$, we mean a single-valued mapping $f: X \rightarrow Y$ such that $f(x) \in F(x)$ for each $x \in X$.

Corollary 2.8. Under conditions of Theorem 2.5, there is a quadratic function $q: X \rightarrow Y$ such that

$$
q(x) \in F(x)+\frac{7 \varepsilon}{3} \overline{B(0,1)} \quad(x \in X) .
$$


Proof. It is known that if $X$ is an abelian group with division by two and $Y$ is a topological vector space, then every subquadratic set-valued function $Q: X \rightarrow$ $C C(Y)$ admits a quadratic selection $q: X \rightarrow Y[4$, Theorem 35.2]. So the result follows from Theorem 2.5.

Acknowledgements. The authors would like to thank the two anonymous reviewers for their helpful comments.

\section{References}

[1] G. Birkhoff, Orthogonality in linear metric spaces, Duke Math. J., 1(1935), 169-172.

[2] J. Brzdȩk, D. Popa, B. Xu, Selection of set-valued maps satisfying a linear inclusion in single variable, Nonlinear Anal. 74(2011), 324-330.

[3] C. Casting and M. Valadier, Convex Analysis and Measurable Multifunctions, Lecture Note in Math. 580(1977).

[4] S. Czerwik, Functional equations and inequalities in several variables, World Scientific Publishing Co. Pte. Ltd (2002).

[5] M. Eshaghi Gordji, S. Abbaszadeh and C. Park, On the stability of generalized mixed type quadratic and quartic functional equation in quasi-Banach spaces, J. Ineq. Appl., 2009(2009), Article ID 153084, 26 pages.

[6] M. Eshaghi and H. Khodaei, Solution and stability of generalized mixed type cubic, quadratic and additive functional equation in quasi-Banach spaces, Nonlinear Anal. 71(2009), 5629?5643.

[7] Z. Gajda and R. Ger, Subadditive mulifunctions and Hyers-Ulam stability, Numer. Math. 80(1987), 281-291.

[8] R. Ger and J. Sikorska, Stability of the orthogonal additivity, Bull. Polish Acad. Sci. Math. 43(1995), 143?151.

[9] D. H. Hyers, On the stability of the linear functional equation, Proc. Nat. Acad. Sci. USA 27(1941), 222-224.

[10] R. C. James, Orthogonality and linear functionals in normed linear spaces, Trans. Amer. Math. Soc., 61(1947), 265?292.

[11] A. K. Mirmostafaee, Approximately additive mappings in non-Archimedean normed spaces, Bull. Korean Math. Soc. 46(2009), No. 2, 387-400.

[12] A. K. Mirmostafaee, Hyers-Ulam stability of cubic mappings in non-Archimedean normed spaces, Kyungpook Math. J. 50(2)(2010), 315-327.

[13] M. S. Moslehian, On the stability of the orthogonal Pexiderized Cauchy equation, J. Math. Anal. Appl., 318(1)(2006), 221-223.

[14] K. Nikodem, On quadratic set-valued functions, Publ. Math. Debrecen 30(1983), 297301.

[15] C. Park, On the stability of the linear mapping in Banach modules, J. Math. Anal. Appl., 275(2002), 711?720. 
[16] C. Park, On the stability of the orthogonally quartic functional equation, Bull. Iran. Math. Soc. 31(1)(2005), 63-70.

[17] D. Popa, A property of a functional inclusion connected with Hyers-Ulam stability, J. Math. Inequal. 4(2009), 591-598.

[18] H. Rådström, An embedding theorem for space of convex sets, Proc. Amer. Math. Soc., 3(1952), 165-169.

[19] J. M. Rassias, The Ulam stability problem in approximation of approximately quadratic mappings by quartic mappings, Journal of Inequalities in Pure and Applied Mathematics, Issue 3, Article 52, 5(2004).

[20] Th.M. Rassias, On the stability of the linear mapping in Banach spaces, Proc. Amer. Math. Soc. 72(1978), 297-300.

[21] J. Rätz, On orthogonally additive mappings, Aequationes Math. 28(1985), 35-49.

[22] J. Sikorska, Generalized orthogonal stability of some functional equations, J. Inequal. Appl. (2006), Art. ID 12404, 23 pp.

[23] A. Smajdor, Additive selections of superadditive set-valued functions, Aequations Math. 39(1990), 121-128.

[24] S. M. Ulam, Problems in Modern Mathematics, Science ed., John Wiley \& Sons, New York, 1964 (Chapter VI, Some Questions in Analysis: Section 1, 